Aus der chirurgischen Universitätsklinik Marburg a. d. Lahn. (Direktor: Professor Dr. A. Läwen.)

\title{
Der Processus vermiformis als Inhalt linksseitiger Hernien.
}

\author{
Von Dr. Karl H. Erb, Assistent der Klinik.
}

Die Beziehungen zwischen Processus vermiformis und Hernie sind in den letzten Jahrzehnten so oft der Gegenstand von kasuistischen Veröffentlichungen und zusammenfassenden Arbeiten gewesen, daß heute wohl für keinen Chirurgen die Appendicitis im Bruchsack und die im Bruch eingeklemmte Appendix noch eine Überraschung bietet. Die Häufigkeit des Vorkommens der Appendix im Bruch geben Hilgenreiner zu I, I Proz., v. Verebelly zu I,8 Proz., Niedlich zu I,4 Proz. an. Zieht man nun in Betracht, daß bei der operativen Autopsie ja eigentlich nur eingeklemmte und verwachsene Hernien ihren wirklichen Inhalt offenbaren, so darf man wohl mit Recht annehmen, daB der Wurmfortsatz ziemlich oft ektopisch liegt und, wie insbesondere $\mathrm{B}$ arsickow und $\mathrm{H}$ on sell nachgewiesen haben, so manche Appendicitis durch diese erhöhte Schädigungsmöglichkeit der Appendix verursacht sein mag.

Ein vor einiger Zeit in der hiesigen Klinik operierter seltener Fall von Vorkommen des Proc. vermif. in einer linksseitigen Hernie gab mir Veranlassung, die in der Literatur verzeichneten diesbezüglichen Fïlle zu sammeln und an das Gefundene Betrachtungen zu knüpfen.

Meist sind derartige Befunde in der Literatur als Curiosa kurz erwähnt, zuweilen finden sie sich in Arbeiten über die Beziehungen zwischen Appendix und Hernien, über Cökalbrüche und über Gleithernien. Mir ist in der Literatur nur eine Arbeit zu Gesicht gekommen, die sich mit dem Viorkommen des Wurmfortsatzes in der linksseitigen Hernie eingehend befaßt, eine Leipziger Dissertation aus dem Jahre I $90 \mathrm{I}$ von $\mathrm{Zach}$ arias, in der der Verfasser einen 
in der Tillmannsschen Klinik operierten und I 8 aus der Literatur zusammengestellte Fälle hinsichtlich der Ät iolog i e des seltenen Krankheitsbildes beleuchtet. Ich habe die mir zur Verfügung stehende Literatur durchsucht und 50 Fälle gefunden, in denen ausdrücklich die Appendix als Inhalt linksseitiger Hernien erwähnt ist. Ich unterscheide zwischen zwei Gruppen von Fällen und führe im folgenden ganz kurze Auszüge aus Krankengeschichten, Zitate und Titelangaben entsprechend gruppiert, an:

A. Fälle, die klinische und anatomische Schlüsse gestatten.

I. B a $\mathrm{z}$ d orf, Berliner klin. Wochenschr. I9I3, S. I 504.

Io Wochen altes Kind. Kongenitale Leistenhernie. Inkarzeration. Inhalt: Cöcum und Appendix.

2. B e cker, Arch. f. Kinderhlkde. 1913, Bd. 59.

2 Monate alter Knabe. Leistenbruch, seit 24 Std. inkarzeriert. Im oberen Wundwinkel die erst nach Hervorziehen erkannte, entzündete und teils gangränöse Appendix. Röntgendurchleuchtung: Herz und Leber an normaler Stelle. Verfasser nimmt bewegliches Cöcum an.

3. Bundschuh, Bruns' Beitr., Bd.3I, S. 48I.

65 j. Mann. Lange bestehender Leistenbruch. Seit $4 \mathrm{~T}$. inkarzeriert. Letzter Stuhl vor $3 \mathrm{~T}$., zuletzt Ileus. Kollaps, Koterbrechen. Bruchinhalt: Cöcum, Wurmfortsatz, $35 \mathrm{~cm}$ Ilelım, etwas injiziert, grebläht; tiefe Schnürfurchen.

4. B rie it er, Bruns' Beitr., Bd. I3.

$62 \mathrm{j}$. Frau mit seit $30 \mathrm{~J}$. bestehender Hernia inguino-properitonealis. $4 \mathrm{~T}$. vor der Einlieferung Inkarzeration. Im Bruchsack fäkulente Flüssigkeit, Cöcum, Appendix, Dünndarmschlinge, Wurm an der Basis perforiert. Exitus beinahe 2 Monate p. op. -

A u topsie: Im wesentlichen Pneumonie, 2 subphrenische Abszesse, geblähter Dickdarm, namentlich Col. transv.; langes Mesocolon, auch des Col. asc. und desc. Col. asc. spitzwinkelig nach unten abgebogen, so daß Cöcum links unten das narbige Peritoneum berührt.

5. Catellani, Annals of Surgery 1898 .

$49 \mathrm{j}$. Frau mit seit $24 \mathrm{~J}$. bestehendem Schenkelbruch. Am Tage vor der Einlieferung Inkarzeration. Sehr hinfälliger Allgemeinzustand. Bruchinhalt: I m Dünnclarm, Col. transv. und asc., Cöcum + Appendix. Colon schwärzlich verfärbt. Serosa bedeckt mit Pseudomembra. nen. Eröffnung der Bauchwand um $5 \mathrm{~cm}$, dann gelingt die Reposition. - Exitus 8 Std. p. op. - A u topsie: Umdrehung des Dünn- und Dickdarmes. Mesocöcum $40 \mathrm{~cm}$, Mesenterium des Dünndarmes $60 \mathrm{~cm}$ lang und sehr dick. $\mathrm{r}^{1 / 2} \mathrm{~m}$ Dünndarm zeigen Inkarzerationsspuren, 
ebenso $80 \mathrm{~cm}$ Cöcum und Colon. Verfasser hält den seltenen Befund nur auf Grund des langen Mesocolons und Mesenteriums für möglich. Es ist seiner Ansicht nach angeboren, nicht erworben.

6. Coley, zit. nach Estor, Revue de Chir. I902.

I j. Knabe mit eingeklemmter Leistenhernie -.- Herniotomie, Abtragung der Appendix. Heilung.

7. D u bar r, zit. nach Es tor, Revue de Chir. 1902.

I6 Monate alter Knabe mit beiderseitiger, kurz nach der Geburt beobachteter Leistenhernie. Finklemmung seit 48 Stunden. - Inhalt: Cöcum mit Proc. vermif. Schnürring am inneren Leistenring.

8. GeiBler, zit. nach $Z$ a charias.

70 j. Frau mit seit $30 \mathrm{~J}$. bestehendem Leistenbruch. Ascites. Täglich mehrmals dünner gelber Stuhl. Exitus. -- A u to p sie: Inhalt der l. Hernie der in der Mitte eingeschnürte, stark wandverdickte Wurmfortsatz. Raum zwischen Symphyse und Nabel durch das Cöcum ausgefüllt, das mit den Därmen und der vorderen Bauchwand verwachsen ist.

9. Gibbon, Annals of Surgery I9oI.

$55 \mathrm{j}$. Mann mit seit $23 \mathrm{~J}$. bestehendem Skrotalbruch. Inhalt: Mehrere Dünndarmschlingen, Cöcum mit Appendix und ein großer Teil des Col. asc. Reposition. Verlauf erst gut. Plötzlich Exitus unter septischen E.rscheinungen. Todesursache: Nierenaffektion?

. Io. Göbel, Deutsche med. Wochenschr. I896, S.46I.

3 Monate alter Knabe. Leistenbruch. Partielles Offenbleiben des Proc. vaginalis peritonei. Bruchinhalt freibeweglicher Proc. vermif. Cöcum drängt sich nach Art eines Darmwandbruches durch den Bruchsackhals in den Bruchsack.

II. Gray, zit. nach $Z$ a charias.

$30 \mathrm{j}$. Mann mit seit $6 \mathrm{~J}$. bestehendem Leistenbruch. Bruchinhalt Cöcum mit Appendix und 3 Zoll Ileum.

12. Griffith, ref. Deutsche med. Wochenschr. I909, S. 220.

Inguinalhernie. Cöcum ließ sich wegen Verwachsungen nicht vorziehen. Geplante Appendektomie fällt daher weg. Wurm wird reponiert.

I3. Habs, Deutsche Zeitschr. f. Chir., Bd. 32, S. 323 .

$52 \mathrm{j}$. Mann mit Leistenbruch. Inhalt angewachsener Wurmfortsatz und degenerierte Appendices epiploicae. Abbinden und Abtragung des Wurms. Verödung des Lumens mit dem Ferrum candens. Kein Situs inversus.

I4. Hieidenreich, zit. nach Niedlich, Bruns' Beitr., Bd. I2I.

$36 \mathrm{j}$. Mann mit seit $4 \mathrm{~J}$. bestehendem Leistenbruch. Inhalt: Cöcum + Appendix.

15. Hildebrand, Deutsche Zeitschr. f. Chir., Bd. 33 .

$25 \mathrm{j}$. Mann. mit seit Jugend bestehender Skrotalhernie. Inhalt: 
Cöcum + Proc. vermif. Darm stark gedehnt, hochrot, dünne Verwachsungen. Reposition der Darmteile.

I6. Hilgen reiner, Bruns' Beitr., Bd. 69, S. 368.

$66 \mathrm{j}$. Frau mit seit ro J. bestehendem Schenkelbruch. Seit $3 \mathrm{~T}$. irreponibel. Okklusionserscheinungen. Bruchinhalt: Unterstes Ileum, Cöcum + Appendix. Cöcum, mit dem Bruchsack innig verwachsen, trägt zwei der Perf. nahe Geschwüre - Resektion von Cöcum und Appendix unter Verlängerung des Bruchschnittes medianwärts bis zur Mitte. Seit-zu-Seit-Anastomose. Kurz vor der beabsichtigten Entlassung nach Erholung Tod an Lungienembolie.

17. vander Hoeven, zit. nach Estor, Revue de Chir. 1902.

I4 Monate alter Knabe mit Leistenbruch. Einklemmungserscheinungen seit $2 \mathrm{~T}$. Inhalt: Dünndarm, Cöcum, Appendix.

I8. Ka newskaja, ref. Zentralbl. f. Chir. I9I4, S. 689.

$38 \mathrm{j}$. Mann mit seit $4 \mathrm{~J}$. bestehendem Leistenbruch. - Hüllen derb, verdickt, überall mit dem Inhalt, dem wandverdickten Cöcum verlötet. Wurm hinten mit dem Blinddarm verwachsen - Repos. des Darmes. Schnitt im rechten Hypogastrium, von hier aus Appendektomie. Da Valvula Bauhini entzündlich infiltriert, Anastomose zwischen Ileum und Col. asc. Wegen zu großer Länge des Mesocöcums Anheftung des Blinddarms ans vordere Bauchfell.

I9. Klose, Bruns' Beitr., Bd.74, S.6oI.

80 j. Mann mit großer Skrotalhernie, die bis zum Knie reicht. Inhalt: Col. asc., Cöcum + Appendix.

20. Koerber, zit. nach Barsickow, Bruns' Beitr., Bd. 68 .

5 I j. Mann mit seit $30 \mathrm{~J}$. bestehendem Leistenbruch. Seit Jahren Schmerzanfälle, vom Bruch in den Leib ziehend. Seit einem Tag Stuhlverhaltung, Winde gehen ab. Kein Erbrechen. Bruch reponibel, stark schmerzhaft. Temp. 37,6. - Bruchinhalt mehrere Dünndarmschlingen, geballtes Cöcum und die stark entzündlich veränderte, mit der Umgebung verwachsene Appendix. Appendektomie Bassini. Am 19. T. Exitus an Pneumonie.

2I. König, zit. nach $Z$ a charias.

$25 \mathrm{j}$. Mann mit seit Jugend bestehendem 'Leistenbruch. Bruchinhalt: Cöcum mit Proc. vermif. Tunica vaginalis testis propria bildet den Bruchsack.

22. Kr ü ger, ref. Zentralbl. f. Chir. I902, S. I054.

8I j. Frau mit Schenkelhernie; Einklemmungserscheinungen. Bruchinhalt ein Stück des Cöcums mit angelagerter Appendix.

23. Markussen, ref. Deutsche med. Wochenschr. I9I I, S. I80.

4 j. Knabe mit Leistenbruch und Einklemmungserscheinungen, Bruchinhalt Cöcum mit entzündeter Appendix, benachbarte Teile des Col. und Ileum. - Verlauf sprach dafür, daß der an normaler Stelle erkrankte Wurmfortsatz erst nach einigen Tagen in den Bruch gelangt war. 
24. Pfister, zit nach $\mathrm{Z}$ acharias.

41 j. Frau mit angeborenem Leistenbruch. Bruchinhalt Col. asc., Cöcum + Proc. vermif. Langgedehntes Mesocol. und Mesocöcum. Eingeweide sehr beweglich.

25. Potherat, zit. nach Lévy, Arch. prov. de Chir. 1903, XII.

Leistenbruch des Cöcum mit komplettem Bruchsacke.

26. Romm, Deutsche Zeitschr. f. Chir., Bd. 4I, S. 249.

$48 \mathrm{j}$. Mann mit I J. altem Schenkelbruche. Seit $4 \mathrm{~T}$. irreponibel. Schmerzhaftigkeit. Kein Erbrechen. Stuhl o. B. Temp. 38,5. Handtellergroße flache Schwellung. Haut entzündlich verändert. Jauchiger penetrant riechender Eiter im Bruchsack. Bruchinhalt Wurmfortsatz mit gangränöser Kuppe und Kotstein. Resektion des Wurmes im Gesunden. Verfasser führt als Grund für die Linkslagerung Länge und Beweglichkeit des Cöcum an.

27. S a rf ert, Deutsche Zeitschr. f. Chir., Bd. 38, S. 29 I.

52 j. Mann mit Skrotalbruch. Pat. kam wegen gleichzeitiger Erkrankung der Lungen und starb unter den Zeichen der Peritonitis. Sektion: In der 1. Skrotalhälfte mehrere Ileumschlingen, Col. asc., Cöcum und Wurm. Därme unter sich verwachsen. Wurm gangränös, an einigen Stellen perforiert. Peritonitis.

28. Spengler, zit nach $Z$ acharias.

$5 \frac{1}{2}$ j. Knabe mit angeborenem Leistenbruch. Seit $1 / 2$ J. Verstopfung, öfters Erbrechen. Schmerzen im Leibe. Oft Repositionsversuche, die nie ganz gelangen. Bruchinhalt ungeheures Konvolut von Därmen mit Cöcum und Proc. vermif. Verwachsungen der dunkelroten Darmteile untereinander und mit dem Bruchsack. Enteritis, Peritonitis. Exitus. Sektion: Fibrinös-eitrige Peritonitis. Col. asc. ganz aus seiner natürlichen Lage gedrängt, und nicht von Därmen bedeckt, sondern auf ihnen gelagert.

29. S s e m e now, ref. Zentralbl. f. Chir. 1910, S. 342.

3 Monate alter Knabe mit seit 2 M. bestehendem Skrotalbruch. Bruchinhalt: Blinddarm. Wurmfortsatz, ein Stück Dünndarm von rechts her zum Blinddarm kommend. Verfasser hält eine Entwick. lungsanomalie, Stillstand der Entwicklung im 3. Fötalmonat, keinen Situs inversus für vorliegend.

3o. Thès e de Tarie 1, zit. nach Es tor, Revue de Chir. 1902.

$2 \mathrm{j}$. Knabe mit Leistenbruch, der bisher reponibel, jetzt eingeklemmt ist. Keine Op. Exitus $15 \mathrm{~T}$. nach Beginn der Inkarzerationserscheinungen. Sektion: $7 \mathrm{~cm}$ Dünndarm und ein Teil des Proc. vermif. als Bruchinhalt.

3I. Za charias, Diss. Leipzig Igor.

$I^{1 / 2} \mathrm{j}$. Knabe mit angeborenem Leistenbruch, seit $3 \mathrm{~T}$. irreponibel. Inhalt: Cöcum mit Appendix. Keine Schnürfurche. - Repos. des wenig bläulichen Darmes nach Erweiterung des Bruchringes. 
32. Zahradnicky, Wiener klin. Rundsch. 1898.

2 j. Knabe mit Leistenbruch von der 6. Lebenswoche ab. Plötzlich Einklemmung infolge krampfartigen Hustenanfalles. Bruchinhalt: Seröses Bruchwasser, Cöcum, Appendix, eine Dünndarmschlinge. Alle drei zeigen Schnürringe. Der Wurm ist Io cm lang, hat ein kurzes Mesenteriolum und verläuft durch den Bruchsackhals in die Bauchhöhle. (Retrograde Inkarzeration nach $\mathrm{M}$ a y d.) Serosa aller eingeklemmten Teile und des peripheren Endes des Wurmfortsatzes leicht injiziert, Col. sigm. an normaler Stelle, Col. asc. läßt sich nach rechts und oben verfolgen. Reposition des Bruchinhaltes. Kein Situs inversus, sondern verzogenes Mesocöcum.

B. Fälle, dienur ganzkurzerwähnt sind bzw. Fälle, die vornehmlich anatomische Schlüsse gestatten.

33. v. A rx, zit. nach $\mathrm{Zach}$ arias.

82 j. Mann mit beiderseitiger Skrotalhernie. Sektion: Starke Kyphose der Brustwirbelsäule, Rippenbogenrand überragt weit das Abdomen. Brüche reichen beiderseits bis zum unteren Oberschenkeldrittel. Im 1. Bruchsack der ganze Dünndarm und hinter ihm Cöcum mit Proc. vermif. und Anfangsteil des Col. asc. Dieses zog weiter durch den rechten Bruch und von da quer durch die Bauchhöhle nach dem 1. Hypochond'rium, um von hier normal, kurz angeheftet als Col. desc. abzusteigen.

34. Bon $\mathbf{n}$, zit nach $\mathrm{Zacharias.}$

Alter Mann mit beiderseitiger I.eistenhernie. Durch starke Linksskoliose der Wirbelsäule und Rechtswendung des Beckens außergewöhnliche Verlagerung des Dickdarmes. In der 1. Hernie lag ein Teil des Ileum mit dem Cöcum und Proc. vermif., sowie ein Teil vom Col. asc., im rechten Bruch Col. transv., Col. desc. und Netz.

35. Bonn, zit. nach $Z$ acharias.

Frau mit Leistenbruch. Sektion: Große Hernie bis zur Mitte des Oberschenkels. Inhalt: Netz, Ileumpartie, Colon, Cöcum mit Appendix, linkes Ovarium, Tube.

36. Cam pier, zit. nach $Z$ acharias.

Alter Mann mit beiderseitigem Leistenbruch. Sektion: In der 1. Hernie Cöcum und Wurm, nicht infolge Transposition der Eingeweide, sondern durch clas Gewicht des Blinddarms dorthin gedrängt.

37. Chanjan, zit. nach $Z$ acharias.

3 Monate altes Kind mit Leistenbruch. Cöcum und Appendix als Bruchinhalt.

38. Corson, zit. nach Lévy, Arch. prov. de Chir. I9o3, XII. Fall von 1. Inguinalhernie, Cöcum und Proc. vermif. als Inhalt. 39. Fogt, zit. nach $Z$ a charias.

Männlicher Pat. mit Skrotalhernie. Sektion: Kindskopfgroße 
Skrotalgeschwulst mit Magen, Netz, Col. transv., Cöcum mit Wurmfortsatz nud einem Stück Ileum als Inhalt.

40. Little, zit. nach Estor, Revue de Chir. 1902.

4 Monate alter Knabe mit Leistenbruch. Inhalt: Cöcum mit Wurmfortsatz und eine Dünndarmschlinge. Langes Mesocöcum.

4I. Méry, zit. nach $Z$ a charias.

Alter Mann mit Leistenbruch. Sektion: Ungeheuer großer Bruch, in der Bauchhöhle nur eine FuBlänge Dünndarm, alle übrigen Darmteile, so auch Cöcum und Appendix, im Bruchsack.

42. Richards, ref. Hildebrands Jahresber. 1909, Bd. I5, S. 1055 .

Leistenbruch. Inhalt: Cöcum + Appendix und Colonteile mit deutlichem Mesenterium.

43. Richards, ref. ebenda. Derselbe Befund wie Fall 42.

44. Rosen berger ref. ebenda. I909, S. 1054.

Inguinalhernie mit Cöcum und Appendix als Inhalt.

45. Russel-Rendler, ref. ebenda. I 908, S.939.

9 Monate altes Kind mit Leistenbruch. Inhalt: Dünndarm, Cöcum und Appendix. Zeichen älterer Entzündung

46. S s e m e now, ref. ebenda. I909, S. I054.

Inkarzerierter Leistenbruch mit Cöcum und Appendix als Inhalt.

47. S s e m e now, ref. ebenda. Derselbe Befund wie Fall 46.

48. Steiger, zit. nach $Z$ a ch arias.

57 j. Frau mit Schenkelbruch. Hernie ohne Sack. Inhalt: Cöcum mit Proc. vermif.

49. S t o i a n of $\mathrm{f}$, ref. Hildebrands Jahresber. 1902, S. 852.

Inkarzerierter Leistenbruch. Inhalt: Cöcum und Appendix.

50. Ten is cheff, zit. nach $\mathrm{N}$ ied lich, Bruns' Beitr. I2I.

Leistenbruch mit Cöcum und Appendix als Inhalt. Heilung.

Anschließend hieran teile ich kurz die Krankengeschichtedes in der Marburger Klinik operierten Falles mit:

$I^{1 / 2}$ j. Knabe 'wurde am 6. IV. I922 mit folgender A n a m nese aufgenommen: Seit der Geburt besteht ein 1. Leistenbruch, der sich merklich vergrößerte, als das Kind zu laufen anfing. Er ließ sich immer ohne Mühe zurückdrängen. Vorgestern fiel den Eltern plötzlich auf, daB er irreponibel geworden war. Seither einmal Erbrechen. Schlechter Appetit, Stuhl weicher als gewöhnlich.

B ef und: Kräftiger Knabe in gutem Ernährnugszustand, Herz und Lungen $\circ$. B. Leib etwas aufgetrieben, aber weich und außer in der 1. Leistengegend offenbar nicht druckempfindlich. Beide Testikel im Scrotum. Über der l. Leistenbeuge besteht eine knapp faustgroße Vorwölbung, über der die Haut gerötet ist. Der Tumor ist von prallelastischer Konsistenz; keine Fluktuation, keine Transparenz, leerer 
Klopfschall. Bei Druck auf die Vorwölbung schreit das Kind. Die Hernie läßt sich deutlich vom Scrotum bis zum inneren Leistenring palpieren, ohne jedoch Schlüsse auf ihren Inhalt zu gestatten. Temp. rektal 37,8. Puls I20, regelmäßig.

Sofortige Operation (Prof. Burckhardt) in Äthernarkose: Freilegung des prall gespannten Bruchsackes, der sich leicht stumpf bis zu seinem ringförmig eingeschnürten Hals freipräparieren läßt. Eröffnung des Bruchsackes, dessen Wand nicht gerade kindlich zart ist. Reichliche Menge klaren Bruchwassers. Den Bruchinhalt bildet das Cöcum mit der leicht gekrümmten, nahezu bis an ihre Spitze mit einem Mesenteriolum versehenen Appendix und eine Ileumschlinge. Alle Darmteile sind rot injiziert, kontrahieren sich aber auf geringe Reize normal. Im Mesenterium des Ileum, der Appendix und im Mesocöcum mehrere bis Bohnengröße geschwollene Drüsen. Abtragung der Appendix, Stumpfversenkung durch Tabaksbeutelnaht, Übernähung mit 3 Lembertnähten. Nach stumpfer Erweiterung des Bruchsackhalsès gelingt die Reposition des Bruchinhaltes ohne Mühe. Abtragung des Bruchsackes, Versorgung seines Stumpfes. Bassininaht. Hautnaht. Komprimierender Verband.

Temp. steigt am nächsten Tage (rektal) auf 38,4 . P. 124 bei gutem Befinden. 8. IV. Temp. normal. Auf Einlauf Stuhl. II. IV. Bisher täglich Stuhl. Bestes Allgemeinbef. I 5. IV. Entfernung der Nähte aus der p.p.i. verheilten Wunde. Röntgenaufnahme nach Wismuteinlauf ergibt normalen Eingeweidesitus. Entlassung als geheilt.

Die Schleimhaut der frisch aufgeschnittenen Appendix zeigte keine Besonderheiten. Patholog.-histologisch fanden sich: Blutaustritte in der Submucosa. Geringe entzündliche lymphocytäre Infiltration. Diagnose: Chronische Appendicitis.

Es stehen mir also insgesamt 5I Fälle zur Beurteilung zur Verfügung. Dabei bin ich mir wohl bewußt, daß bei mancher Cökalhernie und mancher Hernia permagna der Literatur der Proc. vermif. mit im Bruchsack gelegen haben und daß vielleicht noch mancher einschlägige Fall in der mir nicht zugänglich gewesenen, vonehmlich ausländischen Literatur veröffentlicht sein mag. Ich habe mich streng daran gehalten, daß die Appendix als Bruchinhalt ausdrücklich erwähnt ist und z. B. solche Fälle weggelassen, wie den von Snoy, in dem der Verfasser durch die linke Leistenpforte appendektomierte, nachdem bei Zug an dem vorgefallenen Darmstück in der Bruchpforte die Appendix erschien, die bei Beginn der Operation nicht im Bruchsack gelegen hatte. 
Von den 5 I Fällen betrafen 46 inguinale, $5 \mathrm{krurale}$

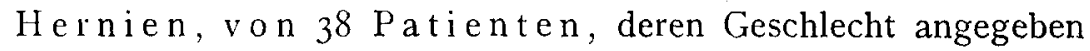
ist, waren $30 \mathrm{männlich,8}$ weiblich. Auf die verschiedenen Lebensalter verteilt sich die linksseitige Appendixhernie folgendermaßen :

I. Lebensjahr 8 Fälle, davon 5 männl. o weibl. 3 fragl. 8 ing. o krur.

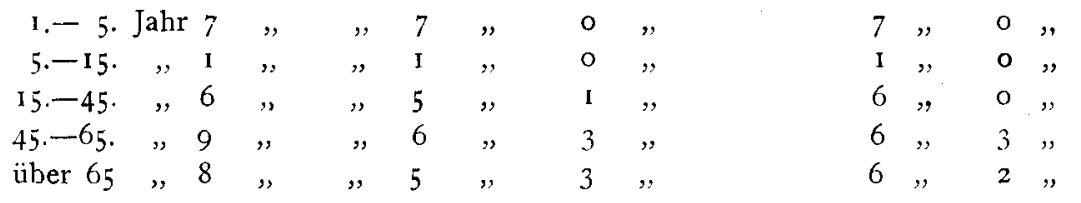

Daraus ergibt sich: hohe Beteiligung des Säuglings- und frühen Kindesalters, sehr geringe Beteiligung des späteren Kindesalters, geringe Beteiligung des besten Arbeitsalters, hohe Beteiligung des höheren Arbeitsalters und des Alters, ein starkes Überwiegen der Männer (im Kindesalter überhaupt keine Mädchen), nennenswerte Beteiligung der Frauen überhaupt erst im Alter. In der Jugend nur Inguinalhernien, Kruralhernien treten gleichzeitig mit der Beteiligung der Frauen in die Erscheinung. Von 8 alten Frauen haben 4 (50 Proz.) Kruralhernien mit Appendix als Inhalt, während nur ein einziger Mann eine solche Kruralhernie hat. Auf die Gründe für diese rein empirischen Beobachtungen werde ich gelegentlich der Ätiologie der Appendixhernie zurückzukommen haben.

Aus der Anamnese der Erkrankungen interessiert zunächst das Alter der Brüche: in I4 Fällen handelte es sich um alte Hernien, die längere Zeit bestanden hatten, 2 mal waren es frisch erworbene, 5 mal kongenitale kindliche Hernien und in einem Fall bestand bei einem $4 \mathrm{I}$ jährigen der Bruch seit der Geburt.

Inkarzerationserscheinungen machten 20 Brüche der Gruppe $A$, bei zweien der Gruppe B ist kurz angegeben, daß es sich um einen eingeklemmten Bruch handelt. Die Einklemmungsererscheinungen bestanden in den meisten Fällen 24 Stunden bis 4 Tage vor der Operation. Zu wirklich schweren Erscheinungen des Darmverschlusses kam es in nur vereinzelten Fällen. Meist beschränkte sich das klinische Bild auf Irreponibilität des Bruches, ganz wenig Erbrechen; in mehreren Fällen wird angegeben, daß der Stuhl häufiger und dünner war als gewöhnlich. 
Dic Weichteilbedeckungen des Bruches waren wiederholt entzündlich verändert. $\mathrm{Zu}$ diesen $\mathrm{im}$ allgemeinen leichten Erscheinungen kam in Fall 2 starke Schmerzhaftigkeit und Temperatur 37,6, im Fall 3 Kollaps, Koterbrechen, starke Druckempfindlichkeit und Meteorismus, im Fall 8 Druckempfindlichkeit, Derbheit des Unterbauches und Ascites, im Fall 5 Meteorismus, Druckempfindlichkeit, hinfälliger Allgemeinzustand, Temperatur 38,o hinzu; doch wird später zu zeigen sein, daß diese Symptome durch besondere pathologisch-anatomische Verhältnisse bedingt waren. In einigen Fällen (18, 20, 28) fällt auf, daß der Bruch schon seit längerer Zeit schmerzhafte Beschwerden macht bzw. verhältnismäßig plötzlich irreponibel geworden ist. Wir werden später sehen, daß dies der Ausdruck einer chronischen Appendicitis im Bruchsack ist.

In der Mehrzahl der Fälle handelte es sich um große Brüche; unter I7 Angaben hierüber finden sich 12, deren Größe mindestens die eines Straußeneies erreicht, 3 mal die eines Hühnereies bis Mannsfaustgröße und nur 2, deren Größe etwa einer Walnuß entspricht. In den Fällen der Gruppe B finden sich mehrere sehr große (Eventerations-)Brüche.

Dic Diagnose auf Mitbteiligung des Wurmfortsatzes ist ante operationem, soweit Angaben darüber vorhanden sind, in keinem der Fälle gestellt worden. Operiert wurden fast sämtliche Fälle der Gruppe A. Die Operation wurde unterlassen in den Fällen 8 (Appendicitis im Bruchsack, Peritonitis), 27 (Lungenerkrankung und Peritonitis) und 30 (Exitus 15 Tage nach Beginn der Inkarzerationssymptome).

In einem Fall der Gruppe B handelte es sich offenbar um einen Gleitbruch. Die kurze Angabe hierüber lautet: Hernie ohne Sack.

Wenden wir uns nun der in dieser Arbeit hauptsächlich interessierenden Frage des Bruchinhalts $z u$, so finden wir Folgendes: Den Bruchinhalt bildeten in II Fällen (3, 4, I I, 16, 17, 29, 31, 32, 40, 45, unser Fall) Cöcum, Appendix und eine Dünndarmschlinge; bei weitem am häufigsten waren Cöcum und Appéndix der Bruchinhalt nämlich in folgenden I9 Fällen: I., 7, IO, I2, I 4, I 5, I 8, 21, 22, 25, 36, 37, 38, 44, 46, 47, 48, 49, 5 ०. Den alleinigen Inhalt des Bruchsackes bildete der Wurmfortsatz nur in 4 Fällen $(2,8$, I 3, 26). Fünf Autoren haben Cöcum und 
Appendix, Col. asc. und Ileumteile im Bruch gefunden (9, 19, 24, 27, 34). Im Falle 20 trat zum vorgefallenen Dünndarm, Cöcum und Appendix noch geballtes Netz hinzu; Spengler (Fall 28) nennt neben Cöcum und Appendix ein ungeheures Konvolut von Därmen, M arkussen (Fall 23) benachbarte Teile des Colon und Ileum, Richards in seinen beiden Fällen $(42,43)$ Colonteile, Fog t (39) Magen, Netz, Col. transv, und ein Stück lleum, Bon n (35) Netz, Ileum, Colon, linkes Ovarium und Tube, Ca tella ni (5) I m Dünndarm, Col. transv. und asc., Thès e de Tariel (30) neben dem Wurmfortsatz $7 \mathrm{~cm}$ Dünndarm, v. A r x (33) hat vor dem im Bruchsack liegenden Cöcum und Colonteilen den ganzen Dünndarm gefunden, Méry (4I) beschreibt eine Eventeration, bei der bis auf I Fußlänge Dünndarm in der Bauchhöhle, alle übrigen Eingeweide in einem linksseitigen Leistenbruch lagen.

In den Fällen 2, 4, 18, 20, 23, 26, 45 und unserem Fall lag eine Appendicitis im Bruchsack vor, davon 4 akute, 4 chronische Fälle. Im Fall 8 handelte es sich um eine typische isolierte Brucheinklemmung der Appendix, offenbar mit konsekutivem Übergreifen der entstandenen Entzündung auf das fixierte Cöcum, und darauffolgender Peritonitis. Hilgenreiners und $\mathrm{Z}$ a hrad n i ck y s Fälle ( 6 und 32) stellen besondere Seltenheiten dar; bei ersterem spielte sich im Bruchsack eine Entzündung des Cöcum mit 2 der Perforation nahen Geschwüren, bei letzterem eine Entzündung des Wurmfortsatzes mit Übergreifen derselben durch das periphere, in der Bauchhöhle gelegene Ende auf das Peritoneum ab; es handelte sich also um einen Schlingenbruch der Appendix mit retrograder Inkarzeration ( $\mathrm{M}$ a y d l).

In 8 Fällen wurden Peritonitiden beobachtet, davon 2 diffuse und 6 im Bruch lokalisierte. Die oben erwähnten Fälle, welche Inkarzerationserscheinungen und überhaupt schwerere Krankheitssymptome darboten, sind fast alle unter den letzterwähnten Fällen von Appendicitis im Bruchsack und Peritonitis enthalten.

Die Operation bestand fast immer in der Herniotomie in Verbindung mit Abtragung des Wurmfortsatzes. In 6 Fällen wurden die Darmteile, auch der Wurmfortsatz, reponiert, in den Fällen 5 und 16 wurde eine Herniolaparotomie ausgeführt, einmal (Fall I8) die links im Bruch gefundene 
Appendix durch einen Laparotomieschnitt im rechten Unterbauch entfernt, das Cöcum durch Naht an der vorderen Bauchwand befestigt und eine Ileokolostomie gemacht. Im Fall i6 mußte Hilgenreiner das Cöcum mit dem Wurmfortsatz resezieren, weil zwei Geschwüre am Cöcum zu perforieren drohten. Er führte dann eine Seit-zu-Seit-Anastomose aus.

In den meisten Fällen der Gruppe A trat Heilung ein. 8 von 32 Fällen verliefen tödlich: Fall 20 wegen Pneumonie, Fall 16 wegen Lungenembolie, nachdem eine entstandene Kotfistel geheilt war, Fall 30 (ohne Angabe des Grundes), Fall 27 wegen alter Lungenerkrankung und hinzugetretener Peritonitis, Fall 8 wegen Peritonitis, Fall 5 aus nicht klarer Ursiache, Fall 4 wegen Pneumonie und zweier subphrenischer Abszesse.

In einer ganzen, Reihe von Fällen finden sich interessante anatomische Mitteilungen der Autoren, die uns Fingerzeige geben hinsichtlich der gleich zu erörterten Frage der Ätiologie des seltenen Krankheitsbildes. In 6 Fällen ist ausdrücklich angegeben, daß kein Situs inversus vorlag, wie dies entweder bei der Operation festgestellt werden konnte oder sich bei röntgenologischer Untersuchung p.o. ergab. 8 Autoren erwähnen, teilweise auf Grund diesbezüglicher Beobachtungen und Messungen, als mutmaßliche Ursache für das Hineingelangen der Appendix in die linke Hernie ein langes Mesocolon und bewegliches Cöcum. In mehreren Fällen nimmt es bei der Größe der Brüche nicht wunder, daß mit vielen anderen Baucheingeweiden (Eventeration) auch Blinddarm und Wurmfortsatz Bruchinhalt geworden sind. In 2 Fällen machen Verbiegungen der Wirbelsäule die Verlagerung des Wurmfortsatzes einigermaßen erklärlich: im Fall 34 kamen sich infolge Rechtsdrehung des Beckens bei gleichzeitiger Linksskoliose gewissermaßen Bruchpforte und Bruchinhalt entgegen; ähnliches bietet der Fall 33, in dem bei hochgrađiger Kyphose der Brustwirbelsäule der Thorax und Oberbauch sich über den Unterleib stülpten und so Verschiebungen der normalen Lage zuwege gebracht haben können. Im Falle io war der Proc. vagin. peritonei bei einem 3 monatigen Kinde offen geblieben, in Fall $2 \mathrm{I}$ bildet die Tunica vagin. propria testis den Bruchsack. Im Fall 23 soll der Verlauf dafür gesprochen haben, daß der an normaler Stelle erkrankte Wurmfortsatz erst nach 
einigen Tagen in den linksseitigen Leistenbruch gelangt ist. Leider war mir Näheres hierüber nicht zugänglich. Vermutlich hat es der Autor aus der Lokalisation des Schmerzes geschlossen, was ja aber bei einem 4 jährigen Kinde nicht als strikter Beweis anzusehen sein dürfte. Sse me noff (Fall 29) hält eine Entwicklungsanomalie, Stillstand auf dem Stande des 3. Fötalmonats, für zugrunde liegend. Camper (Fall 36) gibt an, daß durch Druck von seiten des Dünndarms das Cöcum mit Wurmfortsatz nach links in die Leistenbruchpforte gedrängt worden sei. Im Fall 4 war das Col. asc. spitzwinklig nach unten abgebogen, so daß das Cöcum eine narbige Partie des Peritoneums links unten berührte. In $\mathrm{C}$ at ellanis Fall (5) fand sich bei der Autopsie neben einem $40 \mathrm{~cm}$ langen Mesocöcum und $60 \mathrm{~cm}$ langen Mesenterium eine Umdrehung des Dünn- und Dickdarmes. Im Fall 33 findet sich die Angabe, daß im Bruche das Cöcum mit Proc. vermif. und Col. asc. hintel den Dünndarmschlingen gelegen habe.

Wie ist das Hineingelangen des Wurmfortsatzes in eine linksseitige Hernie nun zu denken? Die meisten Autoren beantworten diese Frage mit der Erklärung durch ein langes Mesocöcum oder Mesocol. asc. und sprechen von beweglichem Ileocökalsegment. L in h a r t behauptet, daB sich das Cöcum in linksseitigen Hernien nur dann findet, wenn es sich um einen enorm großen Bruch handelt, so daß sekundär auch Cöcum prolabieren kann. Schulz spricht von Coloptose, die einerseits angeboren, andererseits bei Erwachsenen erworben sei durch Schnüren, Koprostase und Erschlaffung der Bauchdecken post partum. Gra se r sagt im Handbuch der Chirurgie, daß auf Grund seines langen Mesenteriums die größte Tendenz, Bruchinhalt zu werden, das Ileum etwa $1 / 4 \mathrm{~m}$ oberhalb der Bauhinschen Klappe habe. In den meisten Fällen würden weitere im Bruch gefundene Darmteile als von diesem zuerst vorgefallenen Dünndarm nachgezogen zu betrachten sein; einmal im Bruch liegende Darmteile, die mit der Vergrößerung der Hernie immer weiter hinab treten, erhielten dadurch zwangläufig ein mehr oder weniger stark verlängertes Mesenterium.

Hildebrand hat sich nach Studien an Leichenmaterial eingehend mit der Frage des Zustandekommens von Cökalhernien befaßt und kommt zu der Überzeugung, daß das Cöcum unter 
gewöhnlichen Verhältnissen nicht leicht Bruchinhalt werden kann, daß vielmehr Veränderungen der Größe und Lage desselben hierzu notwendig sind. Hinsichtlich speziell der linken Leistenhernie mit Cöcum als Inhalt ergibt sich für ihn als Ursache Folgendes:

I. Cöcum durch angeborene Anomalie abnorm beweglich und frei, infolge langen Mesocöcums, Mesocolons und abnormer Länge der Ligamente. Es kann dann unter gleichen Bedingungen Bruchinhalt werden wie eine Dünndarmschlinge. Hierher gehören die Brüche, wo im wesentlichen Cöcum und Appendix im Bruchsack liegen.

2. Cöcum durch Zug eines beweglichen Darmteiles oder Netz disloziert und beweglich geworden und diesem $\mathrm{Zug}$ folgend in den Bruch getreten. $\mathrm{Zu}$ dieser Kategorie sind die Fälle zu rechnen, wo sich außer Cöcum noch viel Darm im Bruch befindet, doch kann auch das Cöcum infolge langen Mesocöcums zuerst eingetreten sein und andere Darmteile nach sich gezogen haben. Zum Schluß erwähnt der Verfasser die Möglichkeit eines Situs inversus.

Mit der Frage der linksseitigen Appendixhernie hat sich eingehend bisher nur $Z$ acharias befaßt. Ich benutze seine Einteilung der Momente, die linksseitige Wurmfortsatzhernien begünstigen und füge einige neue hinzu:

I. Vor übergehende Lageveränderungen des Cöcums, hervorgerufen durch verschiedene Füllungszustände desselben.

II. Angeborene Anomalien des Cöcums:

I. Solche, die mit der fötal en Wanderung desselben in Beziehung stehen.

a) Situs inversus abdominalis inf. (Quervain).

b) Die Wanderung ist in der Mittellinie unterbrochen worden.

c) Die Wanderung ist zu weit abwärts gegangen.

2. Es besteht ein sehr langes Mesenterium.

3. Es sind Verwachsungen mit anderen Organen, vor allem mit dem Hoden vorhanden. 
III. Später entstandene Anomalien:

I. Lageveränderungen durch $\mathrm{Zug}$ oder Druck anderer Organe.

2. Koloptose.

3. Veränderungen des Bauchraumesdurch Ver. biegungen des Skeletts.

IV. Besondere Länge des Proc. vermif. bei freier Beweglichkeit.

Engel hat gezeigt, daß das Cöcum von seinem normalen Platz (in der rechten Fossa iliaca unmittelbar auf dem M. psoas, mit der Mittellinie zugewendeten Ende) recht erheblich abweichen kann, ohne jedoch anormal fixiert zu sein. Bei roo Leichen fand er es Io mal in mittlerer Lage, $28 \mathrm{mal}$ hoch über dem Psoas, 30 mal oberhalb der Symphysis oss. pub., 8 mal tief i m B ecken und 4 mal in der Nabelgegend. Ist es luftgebläht, so kann es links bis an die Symphyse gehen, ist es breiig gefüllt, so sinkt es sogar bis in die linke Beckenhälfte.

Über die angeborenen ungewöhnlichen Lagen und Gestaltungen des menschlichen Darmes hat $\mathrm{Koch}$ eingehende Studien angestellt. Er führt unter anderen die Fälle 4, 24, 34 und 4I dieser Arbeit als solche an, bei denen wenigstens mit Wahrscheinlichkeit der Dickdarm hinter dem Dünndarm angenommen werden kann. De Quervain hat dann $\mathbf{I} 902$ über die Rechtslagerung des ganzen Dickdarmes und partiellen Situs inversus gearbeitet und erklärt die Rückwärts- und einseitige Seitwärtsverlagerung sowie die Umkehrung der Dickdarmlage durch Fehlen oder Unvollständigkeit der Nabelschlingendrehung im 2. bis 3. Fötalmonat.

Über die angeborenen Verwachsungen des Cöcums oder besonders des Wurmfortsatzes mit dem Hoden oder dem Peritoneum gingen früher die Ansichten der Autoren auseinander insofern, als von verschiedenen Seiten die Erklärung Rokitanskys durch eine fötale Peritonitis, der sich auch Virchow, Orth u. a. Autoren anschlossen, angezweifelt und angegriffen wurden.

Unter den später entstandenen Anomalien in der Lage des Cöcums habe ich geglaubt, di.e Veränderungen des Bauchraumes und die dadurch bedingte Veränderung der Lage seines Inhalts 
in bezug auf die Wand anführen und in das Schema Zacharias einfügen zu dürfen, die sich ergeben, wenn die Wirbelsäule oder das Becken oder beide sich drehen und biegen. Die Fälle 33 und 34 dieser Arbeit scheinen doch nicht zufälliges Zusammentreffen derartiger Skelettverbiegungen mit Vorkommen von Cöcum und Appendix in linksseitigen Hernien darzustellen.

Wägt man nun Theorie und Praxis gegeneinander ab, so ergibt sich Folgendes:

Zweifellos ist die Appendix schon bei völlig normal gelagertem Cöcum in linksseitige Brüche gelangt, weil eben eine vorübergehende Lageveränderung nach Engel vorgelegen hatte. Hierüber finden sich unter den Fällen keine Angaben, weil naturgemäß der Operateur nichts beobachten konnte, als allenfalls nachher röntgenologisch, daß kein Situs inversus vorgelegen hatte.

Situs inversus ist merkwürdigerweise bisher in keinem einzigen Falle von linkseitiger Appendixhernie festgestellt worden. Immerhin darf man deshalb diese Möglichkeit des Zustandekommens nicht von der Hand weisen, denn zweifellos liegen Beobachtungen über verwandte Hemmungsanomalien, Rückwärtslagerung des Dickdarmes infolge nicht gedrehter Nabelschlinge, vor, wie $\mathrm{Koch}$ es z. B. von unseren Fällen 4, 24, 34, 4I behauptet. Ich füge die Fälle 5, 29 und 33 hinzu. Mühsam und andere Autoren haben die links im Bauch gelagerte entzündete Appendix ektomiert. Warum sollte sie nicht auch einmal bei Situs inversus im Bruch liegen und dort Erscheinungen machen?

Ein sehr langes Mesenterium ist in 8 oben zitierten Fällen ausdrücklich erwähnt. Es scheint wohl sicher, daß die durch langes Mesocöcum oder Mesocolon asc. begründete Verlagerungsmöglichkeit das Cöcum das Hauptkontingent der linksseitigen Cökal- und Appendikularhernien darstellt.

Eine Verwachsung des Cöcum oder Wurmfortsatzes mit dem Hoden ist in keinem unserer Fälle angegeben. Im Fall Io lag ein offengebliebener Proc. vagin. perit. als Bruchsack vor, im Fall 2 I bildete die Tunica vagin. propria testis den Bruchsack. In ersterem Falle lag der Wurm frei beweglich im Bruchsack, im zweiten findet sich darüber keine An- 
gabe. Jedenfalls kann hier das Vorfallen des Proc. vermif. natürlich ohne Zug des Hodens zustande gekommen sein. Die auf fötale Peritonitis zurückzuführenden Verwachsungen zwischen Hoden und Bauchfell einerseits und Cöcum oder Proc. vermif. andererseits scheinen mithin für die Genese der linksseitigen Appendixhernie keine nennenswerte Rolle zu spielen.

Lageveränderung des Cöcum und seines Anhanges durch Zug oder Druck anderer Organe ist sehr häufig. Unsere Zahlen sprechen dafür, daß Graser mit seiner oben zitierten Ansicht recht hat, denn in 26 Fällen war Dünndarm der Begleiter des Cöcums und der Appendix im Bruch. In I9 Fällen lagen Cöcum und Appendix allein ektopisch. Hilgenreiner tritt $\mathrm{Maydl}$ entgegen, welcher behauptet hatte, daß ein reiner Cökalbruch, d. h. ein solcher ohne gleichzeitiges Vorkommen von Dünndarmschlingen, ein sehr seltenes Vorkommnis sei. Er kommt M a y d l insofern entgegen, als er sagt, daß es sich bei alleinigem Vorkommen des Cöcums mit Appendix meist um freie Hernien handelt, die nicht wie inkarzerierte oder verwachsene ihren wirklichen Inhalt offenbaren, da bei horizontaler Lage bei der Vorbereitung zur Operation natürlich ein Teil des Bruchinhalts spon$\tan$ in die Bauchhöhle zurückschlüpfen kann. I ch $\mathrm{muB} \mathrm{Hil-}$ genreiners Ansicht durchaus bestätigen. I 9 der von mir zusammengestellten Fälle weisen nur Cöcum und Appendix als Inhalt auf, aber von diesen 19 waren auch nur 6 eingeklemmt, also eine geringe Anzahl, wenn man bedenkt, da $\beta$ von 32 Fällen dieser Arbeit 20, also 63 Proz., inkarzeriert waren.

Ob die Koloptose, die zur Verlagerung des Cöcums in den Bruch geführt hat, eine angeborene Beweglichkeit dieses Darm. abschnitts oder eine erworbene Erschlaffung des Aufhängeapparates ist, läßt sich weder bei der operativen noch bei der postmortalen Autopsie einwandfrei feststellen. In den oben geschilderterı Fällen finden sich keine klinischen Angaben, ob möglicherweise eine allgemeine Erschlaffung der Haltebänder vorgelegen hat. Es ist aber nicht von der Hand zu weisen, daß ebenso wie eine angeborene Beweglichkeit des Dickdarmes eine nennenswerte erworbene Ptose zur Verschiebung des Cöcums und seines Anhanges in den Bruch führen kann. 
Eine besonders große Länge des Wurmfortsatzes bei freier Beweglichkeit ist $\mathrm{nach}$ unserer $\mathrm{Zusammenstel-}$ lung als Ursache für das Hineingelangen der Appendix in einelinksseitige Hernievonganzuntergeordneter Bedeutung. Den einzigen langen Wurm von Io $\mathrm{cm}$ Länge (bei einem 2 jährigen Kinde!) erwähnt Zahradnicky (Fall 32).

Hinsichtlich der Ätiologie der linksseitigen Appendixhernie sind zweifellos auch die Art der Hernie und das Geschlecht der Patienten von Bedeutung. Hilgenreine r kommt bei der Betrachtung über seine 13 rechtsseitigen Cökalhernien zu dem Resultat, daß es sich ausschließlich um Leistenhernien bei Männern handelt. Dasselbe gilt nach unseren Zahlen für die linke Appendixhernie. 5 kruralen Hernien und 8 Frauen stehen 46 inguinale und 30 Männer gegenüber. Da auch bei uns mit einer Ausnahme die Frauen die Träger der Kruralhernien sind und $\mathrm{H}$ on s e 11 festgestellt hat, daß die Appendix am meisten in Schenkelbrüchen alter Frauen vorkommt, steht doch fest, daß durch die geringe Beteiligung der Frauen die Leistenhernien bei weitem überwiegen.

Hildebrand hat festgestellt, daß bei Kindern das Col. asc. häufig noch in seinem unteren Endefrei b leibt, während bei Erwachsenen nur noch das Cöcum frei ist. Er konnte an Leichenversuchen zeigen, daß bei den meisten Kindern sich das Cöcum leicht an die rechte und auch an die linke Leistenbruchpforte bringen ließ, während dies bei Erwachsenen nur bei gedehntem Zustand des Cöcums möglich war. Daraus ist wohl diestarke Beteiligung des frühen Kindesalters an der linken Appendixhernie zu erklären. Auffallend ist die geringe Beteiligung des späteren Kindesalters (5-I 5 Jahre) und des Alters von I 5-45 Jahren. Nach den Feststellungen von $\mathrm{Clogg}$ (zit. nach $\mathrm{Schnitzer)}$ fand sich in Ioo Leistenhernien bei Kindern $8 \mathrm{mal}$, also in 8 Proz. der Fälle, der Wurmfortsatz, während er nur in I,9 Proz. aller Herniotomien beobachtet wurde.

Bezüglich der Klinik der linksseitigen Appendixhernie ist den Erfahrungen über die rechtsseitige kaum etwas hinzuzufügen. Ich verweise auf die Arbeiten von Barsick ow und 
Honsel1, und aus jüngster Zeit auf die von Niedlich und Otto. Die Diagnosenstellung ist links natürlich noch schwieriger als rechts, weil die Seltenheit des Vorkommens den Untersucher wohl kaum je daran denken läßt, daß die Appendix im Bruch liegen könnte. Bisher ist die Diagnose in keinem Falle gestellt worden. Über die Therapie bestehen heute wohl keine Meinungsverschiedenheiten mehr. Die Appendektomie wird jeder Chirurg ausführen, der den Wurmfortsatz bei der Herniotomie zu Gesicht bekommt, wenn der Zustand des Kranken es irgend erlaubt. Die Prog nos e darf nach den Fällen unserer Zusammenstellung nicht beurteilt werden, da es sich vielfach um alte Fälle aus der Zeit handelt, wo die Appendektomie eine noch nicht so viel geübte Operation war. Sie dürfte wohl der Prognose bei Einklemmung von Dünndarmschlingen gleichzusetzen sein.

\section{Literaturverzeichnis.}

I. Barsickow, Über Appendicitis im Bruchsack. Bruns' Beitr. z. klin. Chir. I9Io, Bd. 68.

2. Engel. Wiener med. Wochenschr. 1857.

3. Gras er. Handbuch d. prakt. Chir., Bd. III, Abschn. 7.

4. Hildebrand, Die Lageverhältnisse des Cöcum und ihre Beziehung zur Entstehung von äußeren Cöcalbrüchen. Deutsche Zeitschr. f. Chir. I 892, Bd. 33 .

5. Hilgen r in er, Seitene und bemerkenswerte Hernien. Bruns' Beitr. z. klin. Chir. 1910, Bd. 69.

6. Honsell, Über die isolierten Brucheinklemmungen des Wurmfortsatzes. Bruns' Beitr. z. klin. Chir. 1903, Bd. 37.

7. Koch, Angeborene ungewöhnliche Lagen und Gcstaltungen des menschlichen Darmes. Deutsche Zeitchr. f. Chir. I 898, Bd. 50.

8. L in ha rt, Vorlesungen über Unterleibshernien, Würzburg I 866.

9. M iu hs a m, Akute Appendicitis bei Situs inversus totalis. Berliner klin. Wochenschr. I9II, H. 30 .

Io. Niedlich, Appendix und Appendicitis im Bruchsack, Bruns' Beitr. z. klin. Chir. 1920, Bd. I 2 I.

If. Otto, Über Appendicitis im Bruchsack. Deutsche Zeitschr. f. Chir. I922, Bd. I70.

12. De Quervain, Uber Rechtslagerung des ganzen Dickdarms und partiellen Situs inversus. Arch. f. klin. Chir. I902, Bd. 65.

13. Schnitzer, Über isolierte Brucheinklemmung des Wurmfortsatzes. Bruns' Beitr. z. klin. Chir. 1909, Bd.62. 
398 ERB, Der Processus vermiformis ais Inhalt linksseitiger Hernien.

I4. Schulz, Über Gleitbrüche und übergroße Hernien. Arch. f. klin. Chir. I912, Bd.98.

15. Snoy, Appendektomie durch die linke Leistenbruchpforte bei normalem Eingeweidesitus. Zentralbl. f. Chir. 1916, S. 748.

16. v. Vérébelly, Über die Komplikationen der Bruchoperation durch den Wurmfortsatz. Bruns' Beitr. z. klin. Chir. 1906, Bd. 48.

17. $\mathrm{Z}$ acharias, Über das Vorkommen des Proc. vermiformis in links. seitigen Hernien. Diss. Leipzig I90I. 